\title{
Comunidades de escorpiones (Arachnida: Scorpiones) del desierto costero transicional de Chile
}

\author{
Communities of scorpions (Arachnida: Scorpiones) \\ of the transitional coastal desert of Chile
}

\author{
PABLO AGUSTO ${ }^{1}$, CAMILO I. MATTONI ${ }^{2}$, JAIME PIZARRO-ARAYA ${ }^{1}$, JORGE CEPEDA-PIZARRO ${ }^{1, *}$ \\ \& FRANCISCO LÓPEZ-CORTÉS

\begin{abstract}
${ }^{1}$ Departamento de Biología, Facultad de Ciencias, Universidad de La Serena, Casilla 599, La Serena, Chile ${ }^{2}$ Division of Invertebrate Zoology, American Museum of Natural History, Central Park West at 79th Street, New York, NY 10024, USA
\end{abstract} \\ * e-mail para correspondencia: jcepeda@userena.cl
}

\begin{abstract}
RESUMEN
En Chile, el orden Scorpiones está representado por 35 especies, distribuidas en las familias Bothriuridae, Iuridae y Buthidae. La mayoría de ellas son elementos endémicos, adaptados a condiciones xéricas. Nosotros estudiamos la estructura taxonómica de la escorpiofauna asociada a las formaciones vegetacionales presentes en el desierto costero transicional de Chile $\left(25-32^{\circ} \mathrm{S}\right)$. Las capturas se realizaron con trampas de intercepción y luz UV. La información de terreno se complementó con antecedentes señalados en la literatura y material de referencia. Identificamos la presencia de nueve especies en un total de 226 especímenes capturados, pertenecientes a las familias Bothriuridae y Iuridae. Los géneros más abundantes de Bothriuridae, representada por ocho especies, fueron Brachistosternus y Bothriurus, con el 55,4\% y el $11 \%$ del total de especímenes capturados, respectivamente. En relación a Brachistosternus, Br. (Leptosternus) roigalsinai fue la especie más abundante, con el 38,9\% del total de los especímenes capturados. Caraboctonus keyserlingi (Iuridae) constituyó el 33,2 \% del total. La mayor riqueza de especies de escorpiones, con seis y siete especies, fueron respectivamente, las formaciones vegetacionales del desierto costero de Huasco $\left(27^{\circ} 52^{\prime} \mathrm{S}\right.$, $\left.71^{\circ} 05^{\prime} \mathrm{O} ; 2^{\circ} 24^{\prime} \mathrm{S}, 71^{\circ} 18^{\prime} \mathrm{O}\right)$ y del matorral estepario costero $\left(29^{\circ} 24^{\prime} \mathrm{S}, 71^{\circ} 18^{\prime} \mathrm{O} ; 30^{\circ} 34^{\prime} \mathrm{S}, 71^{\circ} 42^{\prime} \mathrm{O}\right)$. Los desiertos costero de Tal-Tal $\left(23^{\circ} 52^{\prime} \mathrm{S}, 70^{\circ} 30^{\prime} \mathrm{O} ; 27^{\circ} 51^{\prime} \mathrm{S}, 71^{\circ} 05^{\prime} \mathrm{O}\right)$ y costero de Huasco presentaron especies exclusivas. El análisis de correspondencia mostró que, para la escorpiofauna estudiada, el matorral estepario costero representaría una zona de transición distribucional. Finalmente, discutimos en función de preferencias de sustrato mostradas por algunas de las especies presentes, algunos aspectos relacionados a la ocupación del hábitat.
\end{abstract}

Palabras clave: Scorpiones, Bothriuridae, Iuridae, desierto costero, zonas áridas, Chile.

\begin{abstract}
In Chile, the order Scorpiones has 35 species, included in the families Bothriuridae, Iuridae, and Buthidae. Most of them are endemic species, well adapted to xeric environments. We studied the taxonomic structure of the scorpiofauna with regards to the plant formations present in the transitional coastal desert of Chile $\left(25-32^{\circ}\right.$ S). Captures of scorpions were conducted with pitfall traps and UV light. Data from de field were complemented from literature and reference material. We identified the presence of 9 species in a total of 226 specimens captured, in the families Bothriuridae and Iuridae. The most abundant genera of Bothriuridae, represented by eight species, were Brachistosternus and Bothriurus, with $55.4 \%$ and $11 \%$ respectively of total captured specimens. Regarding Brachistosternus, Br. (Leptosternus) roigalsinai was the most abundant species, with $38.9 \%$ of total captured specimens. Caraboctonus keyserlingi (Iuridae) made the $33.2 \%$ of total captured specimens. The highest species richness of scorpions, with six and seven species were respectively, the plant formations of the coastal desert of Huasco $\left(27^{\circ} 52^{\prime} \mathrm{S}, 71^{\circ} 05^{\prime} \mathrm{W} ; 2^{\circ} 24^{\prime} \mathrm{S}, 71^{\circ} 18^{\prime} \mathrm{W}\right)$ and those of the coastal shrubby steppe $\left(29^{\circ} 24^{\prime} \mathrm{S}, 71^{\circ} 18^{\prime} \mathrm{W} ; 30^{\circ} 34^{\prime} \mathrm{S}, 71^{\circ} 42^{\prime} \mathrm{W}\right)$. The coastal deserts of Tal-Tal $\left(23^{\circ} 52^{\prime}\right.$ $\mathrm{S}, 70^{\circ} 30^{\prime} \mathrm{W} ; 27^{\circ} 51^{\prime} \mathrm{S}, 71^{\circ} 05^{\prime} \mathrm{W}$ ) and Huasco presented exclusive species. The analysis of correspondence showed that, to the scorpiofauna studied, the coastal shrubby steppe would represent a transitional zone of geographic distribution. Finally, we discussed in function of substrate preference, some aspects related to habitat occupation showed by some of the recorded species.
\end{abstract}

Key words: Scorpiones, Bothriuridae, Iuridae, coastal desert, arid zones, Chile. 


\section{INTRODUCCIÓN}

Los escorpiones son un pequeño grupo de artrópodos terrestres que tienen hábitos sigilosos y nocturnos, durante el día se ocultan bajo piedras, troncos y grietas o en galerías que cavan ellos mismos en el sustrato. Tienen una actividad estacional marcada; la mayoría de las especies es estival; la mayor parte del tiempo se encuentran en sus refugios de donde solo salen para alimentarse y reproducirse (Polis 1990, Warburg \& Polis 1990). Se entiende, en general, que los escorpiones son animales estenoicos y poco móviles (Lourenço 1994a, 2002, Prendini 2001). Por su condición de cavadores, muchas especies dependen de la granulometría y humedad del suelo, factores que a su vez están asociados a diferentes tipos de clima y vegetación (Polis 1990, Prendini 2001). Estas características han permitido que los escorpiones se constituyan en modelos biológicos útiles en el análisis de problemas biogeográficos, ecológicos, etológicos y de conservación (Lourenço 1986, 1991, 1994a, 1994b, Polis 1990, Peretti 1997, 2001, Prendini 2001).

El conocimiento de los escorpiones chilenos es escaso. La mayor parte de la información está referida a aspectos sistemáticos (Lourenço 2001, Mattoni 2003, Ochoa 2004, 2005) y taxonómicos, incluyendo catálogos de especies (Cekalovic 1983, Lowe \& Fet 2000), revisiones de géneros (Cekalovic 1966, San Martín \& Cekalovic 1968), redescripciones de especies (Mattoni 2002a, Mattoni \& Acosta 2006) y descripciones de otras nuevas (Cekalovic 1968, 1973, 1974a, 1974b, 1975, 1981, Acosta 1998, Mattoni 2002b, 2002c, Ojanguren-Affilastro 2002, 2003, 2004, 2005, Lourenço 2005, Ojanguren-Affilastro \& Mattoni 2006). De acuerdo a estos antecedentes, la escorpiofauna chilena registra, a la fecha, 35 especies, pertenecientes a las familias Bothriuridae Simon, 1880, Iuridae Thorell, 1876 y Buthidae Koch, 1837 (Cekalovic 1983, Mattoni 2002b, Ochoa 2004, Lourenço 2005, OjangurenAffilastro 2005, Ojanguren-Affilastro \& Mattoni 2006).

Bothriuridae es predominantemente Neotropical y de distribución Gondwánica (Sissom 1990, Prendini 2003). Está representada en el país por los géneros: Bothriurus Peters, 1861, Brachistosternus
Pocock, 1893, Orobothriurus Maury, 1976, Urophonius Pocock, 1893, Tehuankea Cekalovic, 1973, Centromachetes Lönnberg, 1897 y Phoniocercus Pocock, 1893, siendo los últimos tres endémicos de Chile (Cekalovic 1973, 1983, Acosta 1998, Ojanguren-Affilastro 2005). Iuridae presenta una distribución que abarca gran parte de América y Eurasia (Sissom 1990, Sissom \& Fet 2000). En Chile está representada únicamente por el género monoespecífico Caraboctonus Pocock, 1893, siendo endémico para el desierto costero chileno (Ochoa 2005). La familia Buthidae, por su parte está representada por Isometrus maculatus (DeGeer, 1778), especie introducida y registrada solo en la Isla de Pascua (Cekalovic 1983), y por Tityus chilensis Lourenço, 2005, descrita para el extremo norte del país, en la frontera con Bolivia (Lourenço 2005).

El desierto costero transicional de Chile $\left(25-32^{\circ} \mathrm{S}\right)$ representa el límite septentrional del foco de biodiversidad (hotspots) reconocido para Chile Central (Cowling et al. 1996, Gaston 2000); siendo considerado relevante en términos de diversidad, endemismos e interés de conservación biológica para el país (Armesto et al. 1993, Gajardo 1994, Muñoz et al. 1996, Vidiella et al. 1999, Pizarro-Araya \& Jerez 2004, Cepeda-Pizarro et al. 2005a, 2005b). En un estudio sobre distribución de insectos en el desierto de Atacama, Marquet et al. (1998) encontraron que la abundancia y la riqueza de insectos sigue patrones diferentes: mientras la abundancia decreció con la altitud, la riqueza logró su valor máximo a altitudes intermedias, en aparente relación con el patrón de distribución de la vegetación del área de estudio. En la zona costera, las características geomorfológicas (e.g., dunas, cerros, serranías y quebradas), junto a la acción de las neblinas oceánicas, generan hábitats que permiten la existencia de una vegetación que es, en gran parte, ajena a los efectos de las lluvias esporádicas, surgiendo así discontinuidades locales que interrumpen el gradiente vegetacional esperado (Rundel et al. 1990, Squeo et al. 1998). En este trabajo postulamos que la riqueza taxonómica de Scorpiones en el desierto costero transicional de Chile sigue el patrón de distribución de la vegetación. Los objetivos del trabajo fueron: (1) documentar la composición taxonómica de la escorpiofauna 
del desierto costero transicional de Chile y (2) examinar la presencia latitudinal de las especies, especialmente en su relación con las formaciones vegetacionales descritas en el área.

\section{MATERIALES Y MÉTODOS}

\section{Área de estudio y formaciones vegetacionales}

El estudio consideró una transecta latitudinal de aproximadamente $750 \mathrm{~km}$. Esta transecta abarcó

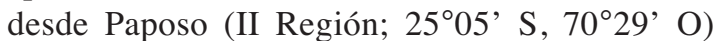

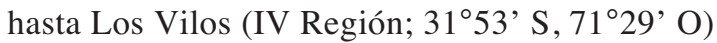
(Tabla 1). El agua que recibe el sistema proviene tanto de lluvias ocasionales como de la incursión de masas de aire húmedo originadas desde el Océano Pacífico (Paskoff \& Manríquez 2004). Esta transecta posee un clima de tendencia mediterránea, con lluvias invernales, de preferencia entre mayo y agosto (Cepeda-Pizarro 1995). De norte a sur existe un gradiente de humedad creciente, con cambios en la cobertura y fisionomía de las formaciones vegetacionales existentes (Gajardo 1994). Estas formaciones corresponden al desierto costero de Tal-Tal (DCTa), desierto costero de Huasco (DCH), matorral estepario costero (MEC), matorral estepario boscoso (MEB), y al matorral estepario arborescente (MEA) (Tabla 1).

\section{TABLA 1}

Localidad de captura o referencia de la escorpiofauna en el desierto costero transicional de Chile $\left(25-32^{\circ} \mathrm{S}\right)$

Collecting sites or references of the scorpiofauna in the transitional coastal desert of Chile $\left(25-32^{\circ} \mathrm{S}\right)$

\begin{tabular}{|c|c|c|c|c|c|}
\hline $\mathrm{N}$ & $\begin{array}{l}\text { Localidad } \\
\text { de captura }\end{array}$ & $\begin{array}{l}\text { Coordenada } \\
\text { geográfica }\end{array}$ & $\begin{array}{l}\text { Altitud } \\
(\mathrm{m})\end{array}$ & $\begin{array}{l}\text { Formación } \\
\text { vegetacional }\end{array}$ & $\begin{array}{l}\text { Método de captura } \\
\text { o referencia }\end{array}$ \\
\hline 1 & Paposo & $25^{\circ} 05^{\prime} \mathrm{S}, 70^{\circ} 29^{\prime} \mathrm{O}$ & 36 & DCTa & Cekalovic (1974a), Lourenço (1995) \\
\hline 2 & Rodillos & $27^{\circ} 05^{\prime} \mathrm{S}, 70^{\circ} 50^{\prime} \mathrm{O}$ & 19 & DCTa & Trampa de intercepción \\
\hline 3 & Carrizal Bajo & $28^{\circ} 05^{\prime} \mathrm{S}, 71^{\circ} 09^{\prime} \mathrm{O}$ & 25 & $\mathrm{DCH}$ & Luz UV \\
\hline 4 & P.N. Llanos de Challe & $28^{\circ} 13^{\prime} \mathrm{S}, 71^{\circ} 04^{\prime} \mathrm{O}$ & 30 & $\mathrm{DCH}$ & Trampa de intercepción, Luz UV \\
\hline 5 & Punta Choros & $29^{\circ} 14^{\prime} \mathrm{S}, 71^{\circ} 28^{\prime} \mathrm{O}$ & 24 & $\mathrm{DCH}$ & Trampa de intercepción \\
\hline 6 & El Temblador & $29^{\circ} 28^{\prime} \mathrm{S}, 71^{\circ} 19^{\prime} \mathrm{O}$ & 20 & MEC & Luz UV \\
\hline 7 & Totoralillo Norte & $29^{\circ} 29^{\prime} \mathrm{S}, 71^{\circ} 19^{\prime} \mathrm{O}$ & 28 & MEC & Luz UV \\
\hline 8 & Caleta Hornos & $29^{\circ} 37^{\prime} \mathrm{S}, 71^{\circ} 16^{\prime} \mathrm{O}$ & 31 & MEC & Luz UV \\
\hline 9 & Porotitos & $29^{\circ} 45^{\prime} \mathrm{S}, 71^{\circ} 20^{\prime} \mathrm{O}$ & 146 & MEC & Luz UV \\
\hline 10 & Punta de Teatinos & $29^{\circ} 49^{\prime} \mathrm{S}, 71^{\circ} 17^{\prime} \mathrm{O}$ & 13 & MEC & Luz UV \\
\hline 11 & Cerro Grande & $29^{\circ} 55^{\prime} \mathrm{S}, 71^{\circ} 13^{\prime} \mathrm{O}$ & 160 & MEC & Luz UV \\
\hline 12 & Lagunillas & $30^{\circ} 06^{\prime} \mathrm{S}, 71^{\circ} 22^{\prime} \mathrm{O}$ & 13 & MEC & Trampa de intercepción \\
\hline 13 & Guanaqueros & $30^{\circ} 12^{\prime} \mathrm{S}, 71^{\circ} 25^{\prime} \mathrm{O}$ & 57 & MEC & Luz UV \\
\hline 14 & Tambillos & $30^{\circ} 12^{\prime} \mathrm{S}, 71^{\circ} 20^{\prime} \mathrm{O}$ & 200 & MEC & Luz UV \\
\hline 15 & P.N. Fray Jorge & $30^{\circ} 37^{\prime} \mathrm{S}, 71^{\circ} 37^{\prime} \mathrm{O}$ & 350 & MEB & Luz UV \\
\hline 16 & Caleta El Limarí & $30^{\circ} 43^{\prime} \mathrm{S}, 71^{\circ} 41^{\prime} \mathrm{O}$ & 15 & MEB & Luz UV \\
\hline 17 & Puerto Oscuro & $31^{\circ} 25^{\prime} \mathrm{S}, 71^{\circ} 35^{\prime} \mathrm{O}$ & 105 & MEA & Luz UV \\
\hline 18 & Ermita Padre Hurtado & $31^{\circ} 28^{\prime} \mathrm{S}, 71^{\circ} 33^{\prime} \mathrm{O}$ & 125 & MEA & Luz UV \\
\hline 19 & Los Vilos & $31^{\circ} 53^{\prime} \mathrm{S}, 71^{\circ} 29^{\prime} \mathrm{O}$ & 28 & MEA & Lourenço (1995) \\
\hline
\end{tabular}

Formaciones vegetacionales: DCTa $=$ desierto costero de Tal-Tal, DCH $=$ desierto costero de Huasco, MEC = matorral estepario costero, $\mathrm{MEB}=$ matorral estepario boscoso, $\mathrm{MEA}=$ matorral estepario arborescente

Plant formations: DCTa $=$ Tal-Tal coastal desert, DCH $=$ Huasco coastal desert, MEC $=$ coastal steppe shrub, MEB = scrubland, MEA = treelike shrubby steppe 
Métodos de captura y sitios de estudio

Este trabajo se basó tanto en registros de literatura (Cekalovic 1974a, 1974b, Lourenço 1995, Ojanguren-Affilastro 2002, 2003, 2004, 2005, Ochoa 2004, 2005, Mattoni \& Acosta 2006), como en capturas realizadas con trampas de intercepción y luz UV (Tabla 1). Las trampas de intercepción se instalaron en sitios representativos de las formaciones vegetacionales de la transecta estudiada. Estos sitios fueron Rodillos $\left(27^{\circ} 05^{\prime} \mathrm{S}, 70^{\circ} 50^{\prime} \mathrm{O}\right)$, Parque Nacional Llanos de Challe $\left(28^{\circ} 13^{\prime} \mathrm{S}\right.$,

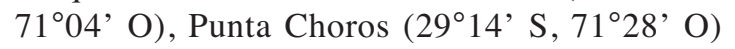
y Lagunillas $\left(30^{\circ} 06^{\prime} \mathrm{S}, 71^{\circ} 22^{\prime} \mathrm{O}\right)$. El funcionamiento y operación de las trampas siguió la metodología descrita por CepedaPizarro et al. (2005a, 2005b). Los muestreos nocturnos con luz UV se llevaron a cabo anualmente entre septiembre y diciembre del período 2000 al 2005 (Tabla 1).

La relación entre los sitios de capturas y las formaciones vegetacionales arriba mencionadas se muestra en la Tabla 1. Los registros fueron cartografiados usando ArcView 3.0 (ESRI 2002). Los límites de las formaciones vegetacionales fueron provistos como archivos de cobertura por el Centro de Estudios Avanzados en Zonas Áridas (CEAZA), La Serena, Chile. Los sitios analizados fueron georreferenciados usando un GPS Garmin ${ }^{\circledR}$, o usando coordenadas provistas por GeoNet Names Server (GNS, http://gnswww.nga.mil/ geonames/GNS/index.jsp).

Para establecer una medida de la asociación de las especies de escorpiones con las distintas formaciones vegetacionales, se realizó un análisis de correspondencia simple (ter Braak 1995), que consideró los valores de las capturas de cada especie en cada formación descritas para la transecta, el análisis se realizó utilizando el programa STATISTICA (StatSoft, Inc. 2001).

El material colectado se determinó a nivel específico siguiendo a Cekalovic (1974a, 1974b), Lourenço (1995), Ojanguren-Affilastro $(2002,2003,2004,2005)$ y Mattoni \& Acosta (2006). Material referencial está depositado en las siguientes colecciones (curadores entre paréntesis); AMNH: American Museum of Natural History, New York, USA (Lorenzo
Prendini); CDA: Cátedra de Diversidad Animal I, Universidad Nacional de Córdoba, Argentina (Luis Acosta); LEULS: Laboratorio de Entomología Ecológica, Universidad de La Serena, Chile (Jorge Cepeda-Pizarro) y MACNAr: Museo Argentino de Ciencias Naturales Bernardino Rivadavia, National Arachnological Collection, Argentina (Cristina Scioscia).

\section{RESULTADOS}

\section{Composición taxonómica de la escorpiofauna}

Solo dos familias, Bothriuridae (Fig. 1 y 2) y Iuridae (Fig. 3) (Tabla 2), estuvieron representadas en los 226 individuos capturados; determinándose la presencia de nueve especies. Bothriuridae representó el $66,8 \%$ del total del material examinado; el resto correspondió a Iuridae $(33,2 \%)$. Dentro de la familia Bothriuridae, encontramos miembros de tres géneros (i.e., Brachistosternus, Bothriurus y Orobothriurus); con respecto a Iuridae, se determinó la presencia de un solo género (i.e., Caraboctonus) (Tabla 2). Los géneros más abundantes de Bothriuridae fueron Brachistosternus y Bothriurus, con el 55,4\% y $11 \%$ del total del material examinado respectivamente. A Orobothriurus le correspondió solo el $0,4 \%$. Para la familia Iuridae, Caraboctonus representó el 33,2 \% del total del material examinado (Tabla 2).

A nivel específico, las especies dominantes fueron Brachistosternus (Leptosternus) roigalsinai Ojanguren-Affilastro, 2002 (38,9\%), Caraboctonus keyserlingi (33,2 \%), Bothriurus coriaceus Pocock, 1893 (7,9 \%) y Br. (L.) artigasi Cekalovic, 1974 (5,8\%) (Tabla 2).

Los sitios de la transecta estudiada que aportaron el mayor número de especies simpátridas fueron Caleta de Hornos (MEC, $\left.29^{\circ} 37^{\prime} \mathrm{S}, 71^{\circ} 16^{\prime} \mathrm{O}\right)$ y Punta Choros $\left(29^{\circ} 14^{\prime}\right.$ $\left.\mathrm{S}, 71^{\circ} 28^{\prime} \mathrm{O}\right)$, con cuatro especies; seguido por el Parque Nacional Llanos de Challe (DCH, $28^{\circ} 13^{\prime} \mathrm{S}, 71^{\circ} 04^{\prime} \mathrm{O}$ ), el Parque Nacional Fray Jorge (MEB, 30³7' S, 71 ${ }^{\circ} 37^{\prime}$ O) y los sectores Punta de Teatinos (MEC, $29^{\circ} 49^{\prime}$ S, $71^{\circ} 17^{\prime}$ O) y Caleta El Limarí (MEB, $30^{\circ} 43^{\prime} \mathrm{S}, 71^{\circ} 41^{\prime} \mathrm{O}$ ), cada uno con tres especies (Tabla 3 ). 


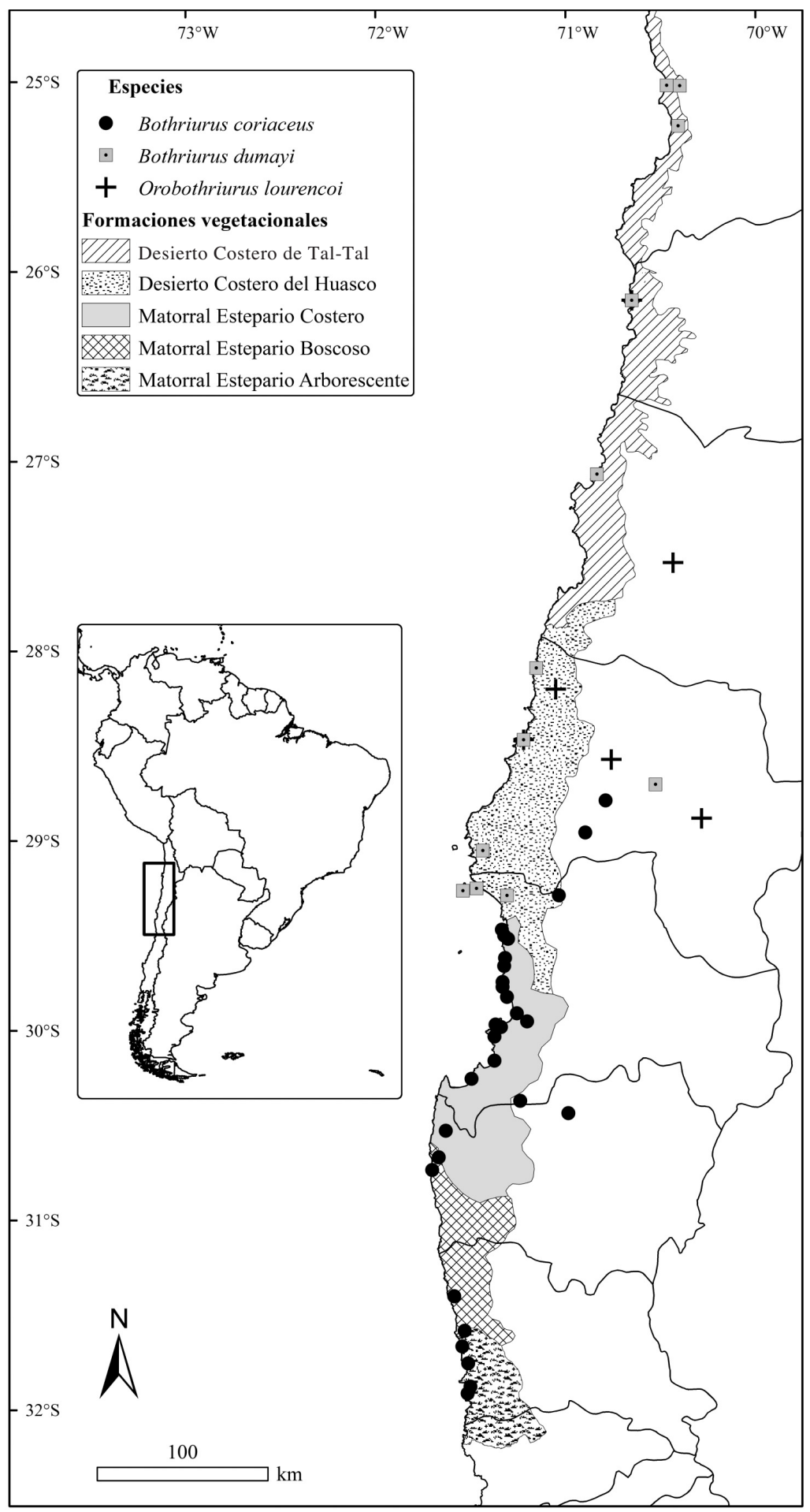

Fig. 1: Distribución geográfica de los géneros Bothriurus y Orobothriurus (Bothriuridae) en el desierto costero transicional chileno $\left(25-32^{\circ} \mathrm{S}\right)$, y formaciones vegetacionales según Gajardo (1994). Datos de este trabajo, Mattoni \& Acosta (2006) y C. Mattoni, L. Prendini \& J. Ochoa (resultados no publicados).

Geographic distribution of the genera Bothriurus and Orobothriurus (Bothriuridae) in the chilean transitional coastal desert (25-32 $2^{\circ}$ ), and plant formations according to Gajardo (1994). Information from this work, Mattoni \& Acosta (2006) and C. Mattoni, L. Prendini \& J. Ochoa (unpublished results). 


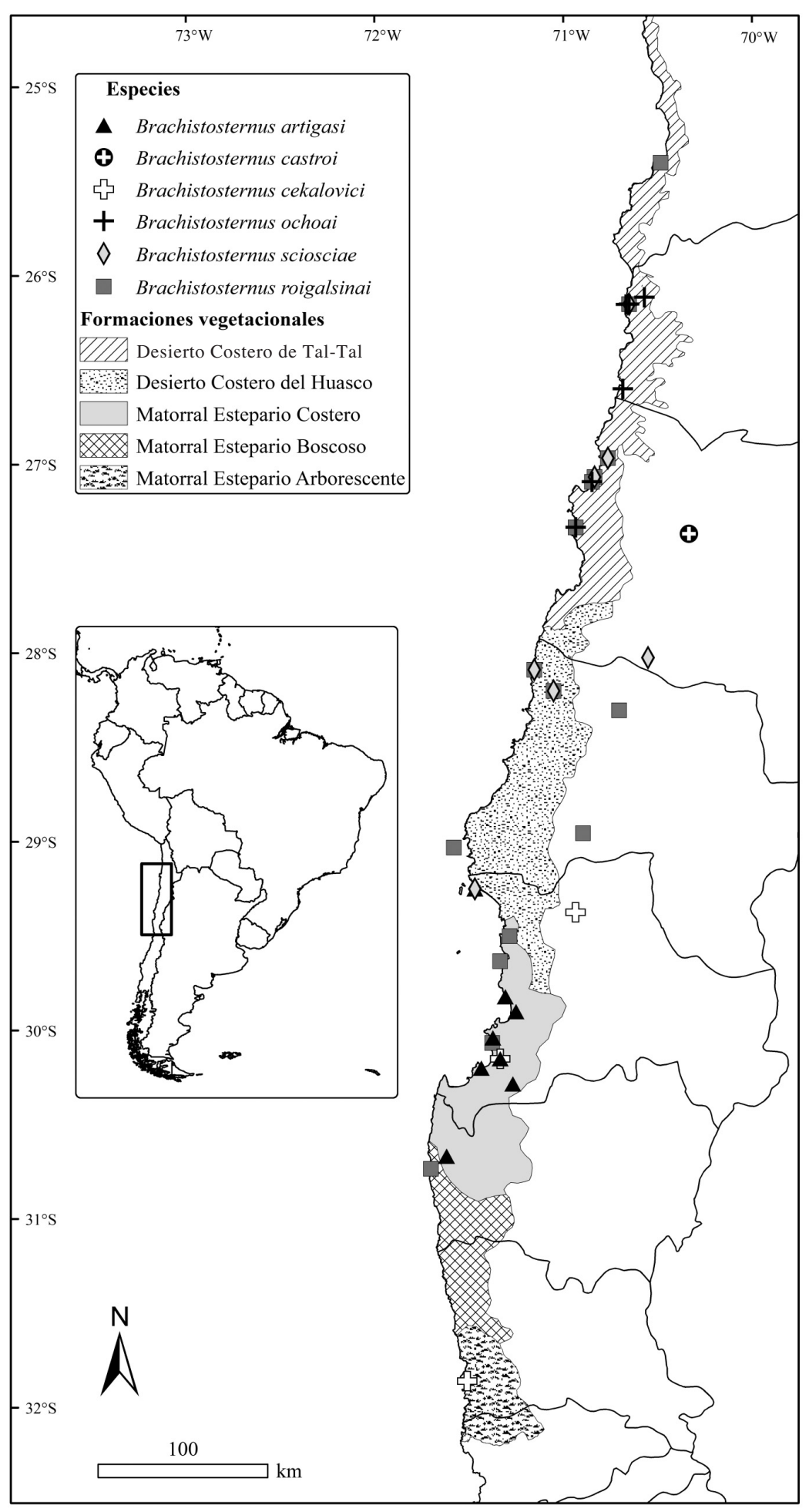

Fig. 2: Distribución geográfica del género Brachistosternus (Bothriuridae) en el desierto costero transicional chileno $\left(25-32^{\circ} \mathrm{S}\right)$, y formaciones vegetacionales según Gajardo (1994). Datos de este trabajo, Cekalovic (1974a) y Ojanguren-Affilastro $(2002,2003,2004,2005)$.

Geographic distribution of the genus Brachistosternus (Bothriuridae) in the chilean transitional coastal desert (25-32 $\mathrm{S}$ ), and plant formations according to Gajardo (1994). Information from this work, Cekalovic (1974a) and Ojanguren-Affilastro $(2002,2003,2004,2005)$. 


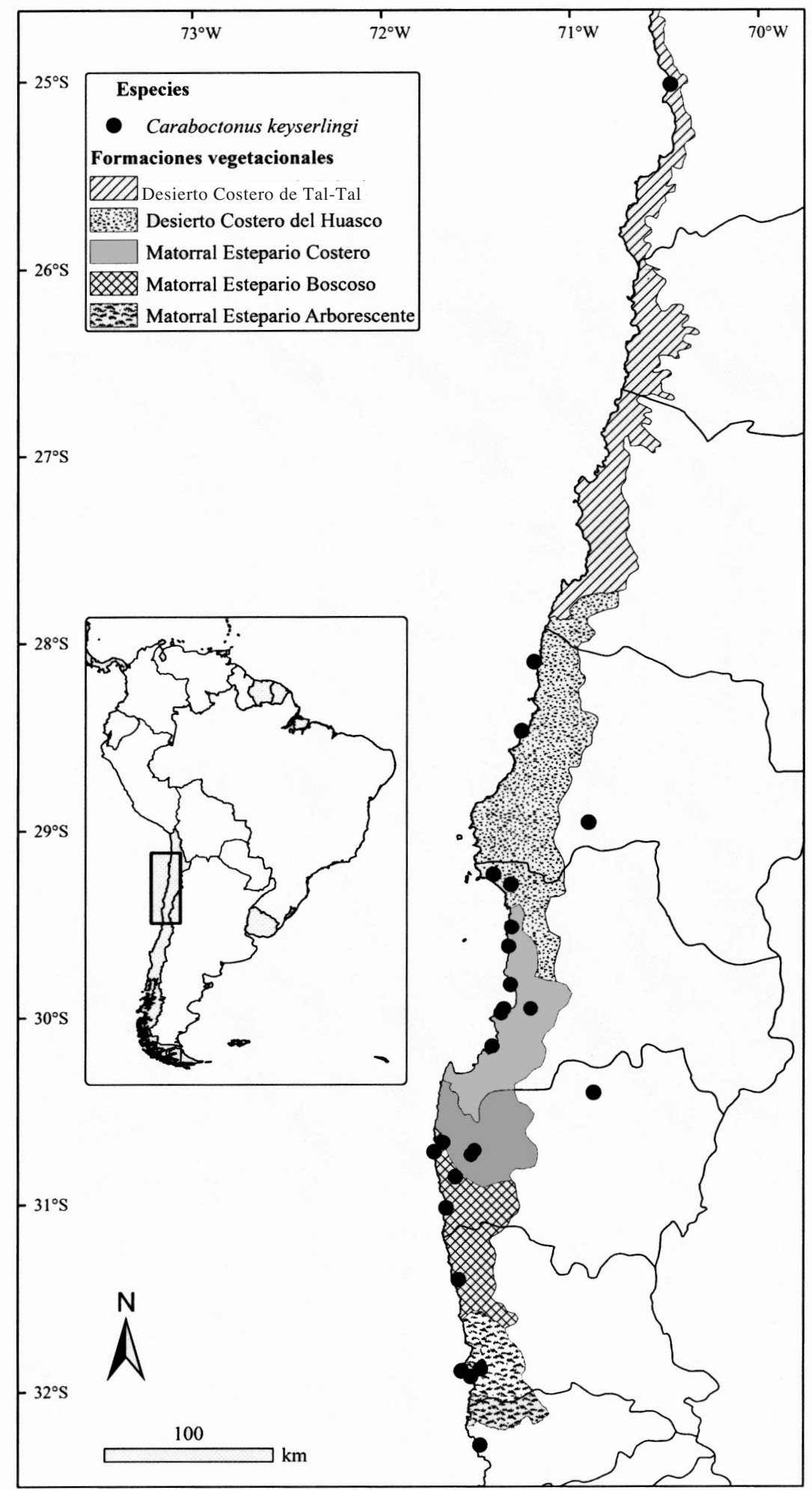

Fig. 3: Distribución geográfica de la familia Iuridae en el desierto costero transicional chileno $\left(25-32^{\circ} \mathrm{S}\right)$. Datos de este trabajo, Lourenço (1995) y C. Mattoni, L. Prendini \& J. Ochoa (resultados no publicados).

Geographic distribution of the Iuridae family in the chilean transitional coastal desert $\left(25-32^{\circ} \mathrm{S}\right)$. Information from this work, Lourenço (1995), and C. Mattoni, L. Prendini \& J. Ochoa (unpublished results). 
TABLA 2

Total de capturas y relaciones porcentuales de la escorpiofauna en el desierto costero transicional de Chile

Captures and percentage relationships of the scorpiofauna in the transitional coastal desert of Chile

\begin{tabular}{llcc}
\hline Familia & Especie & $\mathrm{n}$ & $\%$ \\
\hline \multirow{2}{*}{ Bothriuridae } & Brachistosternus (Leptosternus) roigalsinai & 88 & 38,9 \\
& Brachistosternus (Leptosternus) artigasi & 13 & 5,8 \\
& Brachistosternus (Leptosternus) sciosciae & 11 & 4,9 \\
& Brachistosternus (Leptosternus) cekalovici & 9 & 4 \\
& Brachistosternus (Leptosternus) ochoai & 4 & 7,8 \\
& Bothriurus coriaceus & 18 & 3,1 \\
& Bothriurus dumayi & 7 & 0,4 \\
Iuridae & Orobothriurus lourencoi & 1 & 33,2 \\
Total & Caraboctonus keyserlingi & 75 & 100 \\
\hline
\end{tabular}

Escorpiofauna y formaciones vegetacionales

Las formaciones vegetacionales de la transecta estudiada con mayor riqueza de especies de escorpiones -incluyendo antecedentes bibliográficos (Cekalovic 1974a, 1974b, Lourenço 1995, Ojanguren-Affilastro 2002, 2003, 2004, 2005, Ochoa 2004, 2005, Mattoni \& Acosta en prensa)-, correspondieron al MEC (7) y DCH (6), seguido por el DCTa (4). La menor riqueza correspondió al MEA (2) (Tabla 4).

La familia Iuridae, representada por Caraboctonus keyserlingi, abarcó todo el rango distribucional examinado en este trabajo (Tabla 4). De igual forma, Bothriuridae estuvo representado en las cinco formaciones vegetacionales. Con la excepción de Brachistosternus (Leptosternus) roigalsinai, especie que fue capturada en cuatro de las formaciones vegetacionales (i.e., de norte a sur, DCTa, DCH, MEC y MEB), los otros representantes de Bothriuridae mostraron rangos distribucionales heterogéneos. En este sentido se destacan $B r$. (L.) artigasi, registrada en DCH y MEC; $B r$. (L.) ochoai OjangurenAffilastro, 2004, registrada solo en el DCTa; $B r$. (L.) cekalovici Ojanguren-Affilastro, 2005, registrada solo en el MEC y Orobothriurus lourencoi Ojanguren-Affilastro, 2003, registrada solo en el DCH. La distribución de las especies restantes se muestra en la Tabla 4.

El análisis de correspondencia muestra una fuerte asociación entre especies y formaciones vegetacionales. Las dos primeras dimensiones dan cuenta del 90,3\% de la inercia total $\left(\chi^{2}=\right.$ $252,8 ; \mathrm{P} \ll 0,001)$, reconociéndose un grupo de especies (Br. (L.) artigasi, Br. (L.) cekalovici, B. coriaceus y $C$. keyserlingi), que muestra una fuerte asociación a las formaciones vegetacionales del matorral estepario (i.e., MEC, MEB y MEA). Las especies $B r$. (L.) roigalsinai, $B r$. (L.) sciosciae y $O$. lourencoi se encontraron asociadas a la formación vegetacional del desierto costero de Huasco. Siendo $B r$. (L.) ochoai característica del DCTa, mientras que $B$. dumayi, ocupa una posición transicional, entre DCH y MEC (Fig. 4).

\section{DISCUSIÓN}

Según lo documentado por Polis (1993) para los desiertos de California, Baja California y Namibia, los escorpiones desempeñan un papel clave en los procesos de fragmentación biológica, en los ciclos de los nutrientes y en la dieta de otros organismos consumidores, particularmente vertebrados. Además, forman gran parte de la biomasa total de artrópodos, incluso superando a los vertebrados (Williams 1987). Respecto de la escorpiofauna del desierto costero chileno, esta ha llamado poco la atención de los investigadores chilenos. Gran parte de la información disponible a la fecha es de data antigua, destacando principalmente aspectos de historia natural (Cekalovic 1966, 
TABLA 3

Distribución y composición taxonómica de la escorpiofauna (en términos de ơ', $\$$ y juveniles) en el desierto costero transicional de Chile

Distribution and taxonomic composition of the scorpiofauna (in terms of $\sigma^{\star}, q$ and juveniles) in the transitional coastal desert of Chile

\begin{tabular}{|c|c|c|c|c|c|c|c|c|c|c|c|c|c|c|c|c|c|c|c|c|c|c|c|c|c|c|c|c|c|}
\hline \multirow{3}{*}{$\begin{array}{l}\text { Localidad } \\
\text { de colecta }\end{array}$} & \multirow{3}{*}{$\begin{array}{c}\text { Formación } \\
\text { vegetacional }\end{array}$} & \multicolumn{28}{|c|}{ Especie } \\
\hline & & \multicolumn{3}{|c|}{$\begin{array}{l}B r . \\
(L .) r\end{array}$} & \multicolumn{3}{|c|}{$\begin{array}{l}B r . \\
\text { (L.) } a .\end{array}$} & \multicolumn{3}{|c|}{$\begin{array}{l}B r . \\
\text { (L.) sc. }\end{array}$} & \multicolumn{3}{|c|}{$\begin{array}{l}B r . \\
(L .) c .\end{array}$} & \multicolumn{4}{|c|}{$\begin{array}{c}B r . \\
\text { (L.) och. }\end{array}$} & \multicolumn{3}{|c|}{ B. $c$. } & \multicolumn{3}{|c|}{ B. $d$. } & \multicolumn{3}{|c|}{ O.l. } & \multicolumn{3}{|c|}{ C. $k$. } \\
\hline & & $\sigma^{\prime \prime}$ & 우 & $\mathrm{J}$ & $\sigma^{\prime \prime}$ & 우 & $\mathrm{J}$ & $\sigma^{\prime \prime}$ & 우 & $\mathrm{J}$ & $\sigma^{\prime \prime}$ & 우 & $\mathrm{J}$ & $\sigma^{\prime \prime}$ & ㅇ & $\mathrm{J}$ & & $\sigma^{\prime \prime}$ & 우 & $\mathrm{J}$ & $\sigma^{\prime \prime}$ & 우 & $\mathrm{J}$ & ơ & 우 & $\mathrm{J}$ & $\sigma^{\prime}$ & ㅇ & $\mathrm{J}$ \\
\hline Rodillos & DCTa & 5 & 1 & 1 & & & & & & & & & & 1 & 1 & 2 & & & & & & & & & & & & & \\
\hline Carrizal Bajo & $\mathrm{DCH}$ & 9 & 15 & 4 & & & & 3 & 3 & & & & & & & & & & & & & & & & & & & & \\
\hline $\begin{array}{l}\text { Parque Nacional } \\
\text { Llanos de Challe }\end{array}$ & $\mathrm{DCH}$ & 34 & 5 & 7 & & & & 3 & 1 & & & & & & & & & & & & & & & 1 & & & & & \\
\hline Punta Choros & $\mathrm{DCH}$ & & & & 1 & & 1 & & 1 & & & & & & & & & & & & & 3 & & & & & 1 & 2 & \\
\hline El Temblador & MEC & & & & & & & & & & & & & & & & & 1 & & & & & & & & & & & \\
\hline Totoralillo Norte & MEC & & & & & & & & & & & & & & & & & & & & & 2 & 1 & & & & 3 & 1 & 3 \\
\hline Caleta Hornos & MEC & & 5 & & & & & & & & & & & & & & & 1 & & & & 1 & & & & & 4 & 1 & 4 \\
\hline Porotitos & MEC & & & & & & & & & & & & & & & & & & & 6 & & & & & & & 3 & 3 & 4 \\
\hline Punta de Teatinos & $\mathrm{MEC}$ & & & & 6 & 2 & & & & & & & & & & & & & 2 & & & & & & & & 2 & 3 & 2 \\
\hline Cerro Grande & MEC & & & & & & & & & & & & & & & & & & 1 & & & & & & & & 3 & 6 & \\
\hline Lagunillas & $\mathrm{MEC}$ & & & & 3 & & & & & & 7 & 2 & & & & & & & & & & & & & & & & & \\
\hline Guanaqueros & MEC & & & & & & & & & & & & & & & & & & & & & & & & & & & 3 & 2 \\
\hline Tambillos & MEC & & & & & & & & & & & & & & & & & & & & & & & & & & 3 & 6 & \\
\hline Parque Nacional & & & & & & & & & & & & & & & & & & & & & & & & & & & & & \\
\hline Fray Jorge & MEB & & 1 & & & & & & & & & & & & & & & & 1 & & & & & & & & & 3 & \\
\hline Caleta El Limarí & MEB & & 1 & & & & & & & & & & & & & & & 1 & 4 & & & & & & & & 2 & & 2 \\
\hline Puerto Oscuro & MEA & & & & & & & & & & & & & & & & & & 1 & & & & & & & & 1 & 3 & \\
\hline Ermita Padre Hurtado & MEA & & & & & & & & & & & & & & & & & & & & & & & & & & 1 & 4 & \\
\hline Total & & 48 & 28 & 12 & 10 & 2 & 1 & 6 & 5 & & 7 & 2 & & 1 & 1 & 2 & & 3 & 9 & 6 & & 6 & 1 & 1 & & & 23 & 35 & 17 \\
\hline
\end{tabular}

Clave de especies: Br. (L.) r. = Brachistosternus (L.) roigalsinai, Br. (L.) a. = Brachistosternus $($ L.) artigasi, Br. $($ L.) sc. = Brachistosternus (L.) sciosciae, Br. (L.) c. = Brachistosternus (L.) cekalovici, Br. (L.) och. = Brachistosternus $($ L.) ochoai, B. $c .=$ Bothriurus coriaceus, B. $d .=$ Bothriurus dumayi, O.. . = Orobothriurus lourencoi, $C . k .=$ Caraboctonus keyserlingi Key to species: Br. (L.) r. =Brachistosternus (L.) roigalsinai, Br. (L.) a. = Brachistosternus $(L$.$) artigasi, Br. (L$.) sc. $=$ Brachistosternus (L.) sciosciae, Br. (L.) c. = Brachistosternus (L.) cekalovici, Br. (L.) och. = Brachistosternus (L.) ochoai, B.. . = Bothriurus coriaceus, B. d. = Bothriurus dumayi, O. l. = Orobothriurus lourencoi, C. . $=$ Caraboctonus keyserlingi

1968, 1973, 1974a, 1974b, 1975, 1981, San Martín \& Cekalovic 1968). Investigaciones recientes han incrementado la riqueza de especies para el área y ampliado los rangos distribucionales de varias de ellas (Acosta 1998, Mattoni 2002a, 2002b, 2002c, 2003, Ojanguren-Affilastro 2002, 2003, 2005, Ochoa 2004, 2005, Lourenço 2005, OjangurenAffilastro \& Mattoni 2006).

Estos antecedentes han mejorado la comprensión acerca del origen y distribución del orden en Sudamérica y su relación con el desierto chileno (Mattoni 2003, Ochoa 2005, OjangurenAffilastro 2005). Los actuales patrones distribucionales de la escorpiofauna sudamericana serían producto de la situación geográfica y de los cambios geomorfológicos, climáticos y ecológicos ocurridos en esta parte del continente desde el Terciario (Lourenço 1994b). Sin embargo, estos antecedentes no permiten explicar la presencia de elementos endémicos en los desiertos costeros del norte de Chile. Estos desiertos corresponden a franjas longitudinales que representan zonas climáticas, ecológicas y geomorfológicas diferentes (Abreu \& Bannon 1993). El desierto costero transicional del norte de Chile, debido a su ubicación y a su temprano aislamiento geográfico (ca, 48 a 25 millones aP) (Shmida 1985), mantiene una fauna de artrópodos altamente endémica (Pizarro-Araya \& Jerez 2004, Cepeda-Pizarro et al. 2005b, Flores \& PizarroAraya 2006), con especies de características 
primitivas (e.g., Elasmoderus Saussure, 1888 (Orthoptera), Cnemalobus Guérin-Méneville, 1838 (Carabidae) y Acanthogonatus Karsch, 1880 (Nemesiidae) (Roig-Juñent \& Flores 2001, RoigJuñent et al. 2006). Sin embargo, en el caso de los escorpiones, la mayoría de las especies actuales representan grupos derivados, adaptados a las condiciones crecientes de aridez (Prendini 2003). Por otra parte, una especie ampliamente distribuida en el desierto costero transicional chileno, Caraboctonus keyserlingi, representa evidentemente un elemento muy antiguo, estando los otros miembros de la Familia Iuridae presentes en Perú, Ecuador, México, Estados Unidos, Grecia y Turquía, lo que supone una diversificación de la familia muy antigua, cercana a la separación de Pangea (Sissom \& Fet 2000).

Para la transecta de estudio, se tiene registro de nueve especies de escorpiones; la mayoría de ellas presenta distribución geográfica restringida a una (e.g., Br. (L.) cekalovici, $B r$. (L.) ochoai, O. lourencoi) o dos de las formaciones vegetacionales estudiadas en este trabajo (e.g., Br. (L.) artigasi, Br. (L.) sciosciae); los géneros Brachistosternus y Bothriurus tienen un probable origen o diversificación reciente (10 millones aP), en respuesta a la aridización de esta parte del continente; pero como mencionamos antes, otros componentes, tales como Caraboctonus keyserlingi y Orobothriurus lourencoi, quizás representen elementos mucho más antiguos.

Según los registros taxonómicos disponibles a la fecha, Bothriuridae -representado en el área por los géneros Brachistosternus, Bothriurus y Orobothriurus-, es la familia más especiosa y abundante del desierto costero transicional de Chile. Brachistosternus es el género más diverso del país, con 13 especies endémicas (OjangurenAffilastro 2004, 2005, Ojanguren-Affilastro \& Mattoni 2006). Cinco de ellas tienen registros en el área de estudio: $B r$. (Leptosternus) roigalsinai, $B r$. (L.) sciosciae, Br. (L.) cekalovici, Br. (L.) artigasi y $B r$. (L.) ochoai. Aparentemente, las especies de Brachistosternus muestran una mayor tolerancia interespecífica, siendo posible

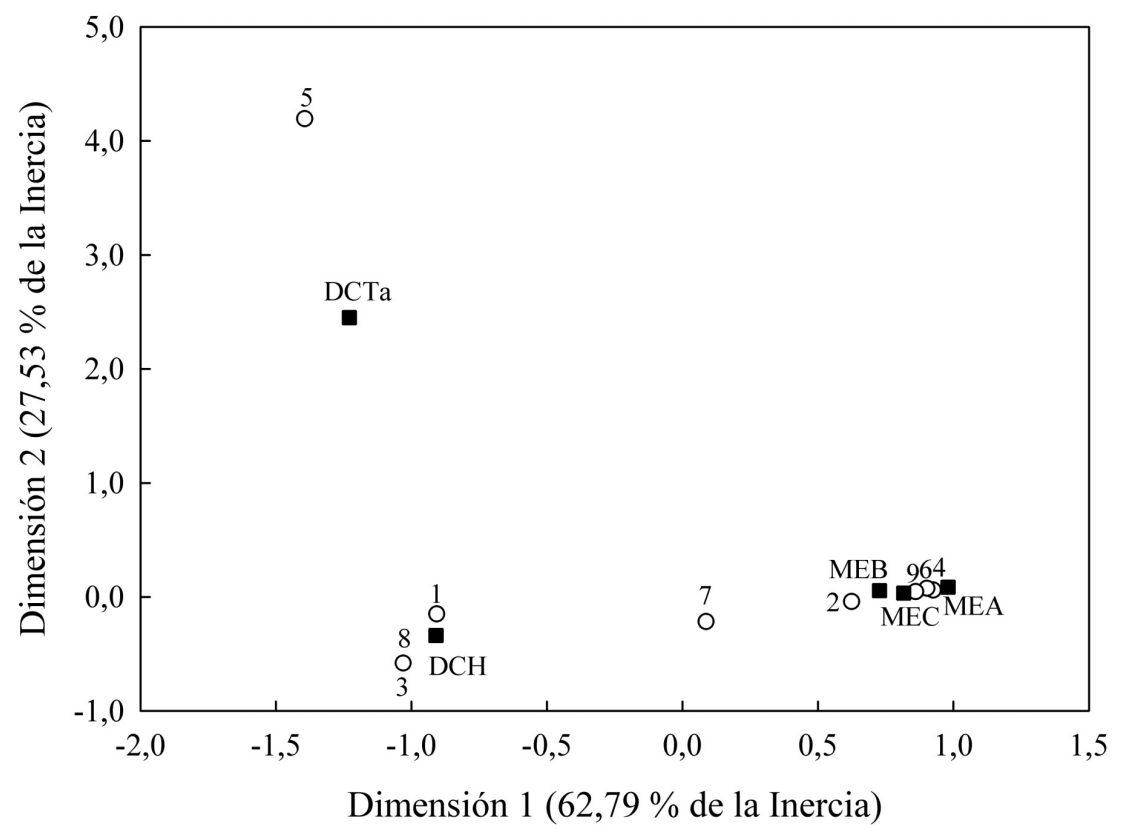

Fig. 4: Representación gráfica de dos dimensiones del análisis de correspondencia entre las formaciones vegetacionales $(\square)$ y las especies de escorpiones $(\mathrm{O})$. Especies: $1=B r$. $(L$. $)$ roigalsinai, $2=$ $B r$. (L.) artigasi, $3=B r$. (L.) sciosciae, $4=B r$. (L.) cekalovici, $5=B r$. (L.) ochoai, $6=B$. coriaceus, $7=B$. dumayi, $8=O$. lourencoi, $9=C$. keyserlingi. Formaciones vegetacionales: DCTa $=$ desierto costero de Tal-Tal, $\mathrm{DCH}=$ desierto costero de Huasco, MEC = matorral estepario costero, $\mathrm{MEB}=$ matorral estepario boscoso, $\mathrm{MEA}=$ matorral estepario arborescente.

Two dimensional graph of the equivalence analysis between the plant formations $(\mathbf{\square})$ and the scorpion species $(\mathrm{O})$. Species: $1=B r$. (L.) roigalsinai, $2=B r$. (L.) artigasi, $3=B r$. (L.) sciosciae, $4=B r$. (L.) cekalovici, $5=B r$. (L.) ochoai, 6 $=B$. coriaceus, $7=B$. dumayi, $8=O$. lourencoi, $9=C$. keyserlingi. Plant formations: DCTa $=$ coastal desert of Tal-Tal; $\mathrm{DCH}=$ coastal desert of Huasco; $\mathrm{MEC}=$ coastal shrubby steppe; $\mathrm{MEB}=$ scrubland MEA = treelike shrubby steppe. 
encontrar en casi todos los sitios de estudio especies simpátricas. Algunas varían en su preferencia de microhábitat, como $B r$. (L.) roigalsinai y $\mathrm{Br}$. (L.) sciosciae, que son generalmente encontradas en simpatría. Sin embargo, observaciones más detalladas muestran que ellas difieren en sus requerimientos de sustratos: $B r .(L$.) roigalsinai prefiere suelos más estables y de mayor granulometría que $B r$. $(L$.) sciosciae, la cual es más común en suelos más inestables y de textura más fina (C. Mattoni, A. Ojanguren-Affilastro, L. Prendini \& J. Ochoa, resultados no publicados). Bothriurus es el segundo género más diverso del país, registrando a la fecha ocho especies, todas endémicas (Lowe \& Fet 2000, Ojanguren-Affilastro 2002, 2003, Mattoni 2003). En la transecta de estudio, el género está representado por $B$. coriaceus y $B$. dumayi. Estas dos especies son morfológicamente similares y prefieren suelos estables, con algún tipo de vegetación. Se registró simpatría de estas especies solamente en una de las nueve localidades de MEC (i.e., Caleta de Hornos), lo cual probablemente sugiere exclusión ecológica
(C. Mattoni, L. Prendini \& J. Ochoa, resultados no publicados). Nuestros resultados muestran que Bothriurus dumayi se distribuye en el área desde el DCTa hasta el MEC. Por el contrario, Bothriurus coriaceus tiene una distribución costera más meridional, aunque esta especie también ha sido registrada en localidades del centro de Chile, incluso en altitudes cercanas a los $1.400 \mathrm{~m}$ (Mattoni \& Acosta 2006). Orobothriurus es un género que agrupa a especies de distribución mayoritariamente andina (Maury 1976, Ochoa 2004); O. lourencoi es la única especie del género adscrita al país y la primera registrada a nivel del mar (Ojanguren-Affilastro 2003, C. Mattoni, L. Prendini \& J Ochoa, resultados no publicados). En la transecta de estudio se encontró exclusivamente en el Parque Nacional Llanos de Challe (DCH, 28 $8^{\circ} 3^{\prime} \mathrm{S}$, $\left.71^{\circ} 04^{\prime} \mathrm{O}\right)$. Esta especie está muy ligada al hábitat pedregoso, donde prefiere laderas coluviales, siendo la única especie de escorpión presente en este tipo de ambiente (Ojanguren-Affilastro 2003, C. Mattoni, L. Prendini \& J. Ochoa, resultados no publicados).

TABLA 4

Distribución de especies de escorpiones en relación a formaciones vegetacionales del desierto costero transicional chileno

Distribution of scorpion species in relation to plant formations present in the transitional coastal desert of Chile

\begin{tabular}{|c|c|c|c|c|c|c|c|}
\hline \multirow{2}{*}{$\begin{array}{l}\text { Taxon } \\
\text { Familia }\end{array}$} & \multirow[b]{2}{*}{ Especie } & \multicolumn{5}{|c|}{ Formación vegetacional } & \multirow[t]{2}{*}{ Fuente } \\
\hline & & DCTa & DCH & MEC & MEB & MEA & \\
\hline \multirow[t]{13}{*}{ Bothriuridae } & Brachistosternus & & & & & & \\
\hline & (L.) roigalsinai & + & + & + & + & & Ojanguren-Affilastro $(2002,2005)$, LEULS \\
\hline & Brachistosternus & & & & & & \\
\hline & (L.) artigasi & & + & + & & & Cekalovic (1974a), LEULS \\
\hline & Brachistosternus & & & & & & \\
\hline & (L.) sciosciae & & + & + & & & Ojanguren-Affilastro $(2002,2005)$, LEULS \\
\hline & Brachistosternus & & & & & & \\
\hline & (L.) cekalovici & & & + & & & Ojanguren-Affilastro (2005), LEULS \\
\hline & Brachistosternus & & & & & & \\
\hline & (L.) ochoai & + & & & & & Ojanguren-Affilastro (2004), LEULS \\
\hline & Bothriurus coriaceus & & & + & + & + & LEULS \\
\hline & Bothriurus dumayi & + & + & + & & & Cekalovic (1974b), LEULS \\
\hline & Orobothriurus lourencoi & & + & & & & Ojanguren-Affilastro (2003), LEULS \\
\hline Iuridae & Caraboctonus keyserlingi & + & + & + & + & + & LEULS \\
\hline Total & & 4 & 6 & 7 & 3 & 2 & \\
\hline
\end{tabular}

Abreviaturas: LEULS = Laboratorio de Entomología Ecológica de la Universidad de La Serena Abbreviations: LEULS = Laboratory of Ecologic Entomology of the University of the La Serena 
Iuridae es una familia de distribución cosmopolita, representada en Sudamérica por los géneros Hadruroides y Caraboctonus, estando este último representado en Chile por $C$. keyserlingi, especie endémica que se distribuye desde Paposo hasta Santiago (Lourenço 1995). Esta especie muestra variaciones morfológicas respecto de la distribución de las tricobotrias it e $i b$ de la cara interna de la pinza y la disposición de las $e b(1$ a 5) de la cara externa de la tibia. Aparentemente, esta variación sigue una tendencia norte-sur (Lourenço 1995). C. keyserlingi se registró en todas las formaciones vegetacionales consideradas en este trabajo. Capturas UV mostraron que la especie es común en las lomas costeras del Parque Nacional Llanos de Challe (C. Mattoni, L. Prendini \& J. Ochoa, resultados no publicados). Esta especie también ha sido capturada en sitios del secano interior del semidesierto chileno (a más de $1.300 \mathrm{~m}$ de altitud), tanto en suelos naturales como cultivados (e.g., plantaciones de Eucalyptus globulus) (C. Mattoni, J. Ochoa \& J. Pizarro-Araya, resultados no publicados).

En la subregión del matorral estepario, se encontró el mayor número de especies de escorpiones asociadas y con los mayores valores de frecuencia de captura. Por su parte MEC, correspondió a una formación vegetacional de carácter ecotonal, constituyendo una transición en la distribución latitudinal de $B r$. (L.) artigasi y B. dumayi. Además, mostró los mayores valores de frecuencia de captura de $C$. keyserlingi, y reunió a $B r$. (L.) cekalovici, especie exclusiva de esta formación. De las especies asociadas al DCH, Br. (L.) sciosciae y $O$. lourencoi son exclusivas de esta formación, como lo es $B r$. (L.) ochoai del DCTa.

Las formaciones vegetacionales MEC y DCH presentaron la mayor riqueza de especies. Referente al DCH, estos antecedentes concuerdan con lo informado por Armesto et al. (1993) respecto de la diversidad de geófitas y hemicriptófitas y por Pizarro-Araya \& Jerez (2004) respecto de la diversidad de Tenebrionidae (e.g., Gyriosomus). Estos resultados apoyan la idea que el desierto costero de Huasco puede ser un foco de diversidad de especies interrumpiendo la gradiente esperada. Discontinuidad que también ha sido documentada para otros sitios del desierto chileno (Marquet et al. 1998, Rau et al. 1998, Squeo et al. 1998). Para el caso particular del desierto costero de Huasco, no se dispone aún de una explicación aceptable. Aparentemente, la presencia de ambientes dunarios estabilizados tendrían un papel determinante en la existencia de estos focos de diversidad biótica, particularmente con animales de hábitos édafo-epígeos específicos.

En términos de abundancia, destacaron las especies Brachistosternus (Leptosternus) roigalsinai y Caraboctonus keyserlingi, con el 38,9 y $33,2 \%$ del total capturado, respectivamente. Gran parte de los especímenes de $B r$. (L.) roigalsinai correspondió a material capturado en el Parque Nacional Llanos de Challe (DCH) en 1997 (año-ENOS intenso, Cepeda-Pizarro et al. 2005a). Este fue un año de abundancia de insectos, particularmente de tenebriónidos. El elevado número de $B r$. (L.) roigalsinai podría estar relacionado con esta mayor disponibilidad de alimento, según ha sido observado en desiertos de otras latitudes (Polis 1980, 1990). De lo anterior se desprende la importancia que puede jugar el evento ENOS en la dinámica comunitaria de los artrópodos terrestres asociados al sistema en estudio (Deslippe et al. 2001, Cepeda-Pizarro et al. 2005a, 2005b, Frankie et al. 2005).

\section{AGRADECIMIENTOS}

Agradecemos a CONAF (III y IV Región) por otorgar las facilidades para trabajar en los Parques Nacionales Llanos de Challe y Fray Jorge. A Víctor Fet (Marshall University, USA), José A. Ochoa (Museo de Historia Natural de la Universidad San Antonio Abad del Cuzco, Perú), Mario Elgueta (Sección Entomología, Museo Nacional de Historia Natural, Chile) y Lorenzo Prendini (American Museum of Natural History, USA), por la ayuda en la obtención de literatura. A José A. Ochoa por las valiosas sugerencias realizadas al manuscrito original. A Andrés Ojanguren-Affilastro (Museo Argentino de Ciencias Naturales Bernardino Rivadavia, Argentina), por los comentarios al manuscrito y por información sobre Brachistosternus. A David López Aspe (Centro de Estudios Avanzados en Zonas Áridas (CEAZA), La Serena, Chile) por proveernos las coberturas SIG de las formaciones vegetacionales. Este trabajo 
fue financiado por el proyecto DIULS $\mathrm{N}^{\circ}$ 220.2.17 (J.P.A.), Dirección de Investigación, Universidad de La Serena, La Serena, Chile.

\section{LITERATURA CITADA}

ABREU ML \& PR BANNON (1993) Dynamics of the South American coastal desert. Journal of the Atmospheric Sciences 50: 2952-2964.

ACOSTA LE (1998) Urophonius transandinus sp. nov. (Bothriuridae), a scorpion from Central Chile. Studies on Neotropical Fauna and Environment 33: 157-164.

ARMESTO JJ, PE VIDIELLA \& JR GUTIÉRREZ (1993) Plant communities of the fog-free coastal desert of Chile: plant strategies in a fluctuating environment. Revista Chilena de Historia Natural 66: 271-282.

CEKALOVIC T (1966) Contribución al conocimiento de los escorpiones chilenos. Noticiario Mensual del Museo Nacional de Historia Natural (Chile) 10: 1-8.

CEKAlOVIC T (1968) Phoniocercus sanmartini, una nueva especie de Bothriuridae de Chile (Scorpionida-Bothriuridae). Boletín de la Sociedad de Biología de Concepción (Chile) 40: 27-32.

CEKALOVIC T (1973) Tehuankea moyanoi n. gen. y $\mathrm{n}$. sp. de escorpión chileno (Scorpiones, Bothriuridae). Boletín de la Sociedad de Biología de Concepción (Chile) 46: 41-51

CEKALOVIC T (1974a) Dos nuevas especies chilenas del género Brachistosternus (Scorpiones, Bothriuridae). Boletín de la Sociedad de Biología de Concepción (Chile) 47: 247-257.

CEKALOVIC T (1974b) Bothriurus dumayi n. sp. de escorpión chileno (Scorpiones, Bothriuridae). Boletín de la Sociedad de Biología de Concepción (Chile) 48: 209-216.

CEKALOVIC T (1975) Brachistosternus (Leptosternus) negrei $\mathrm{n}$. sp. de escorpión de Chile (Scorpiones, Bothriuridae). Brenesia 6: 69-75.

CEKALOVIC T (1981) Dos nuevas especies y un nuevo registro del género Urophonius para Chile (Scorpiones, Bothriuridae). Boletín de la Sociedad de Biología de Concepción (Chile) 52: 195-201.

CEKALOVIC T (1983) Catálogo de los escorpiones de Chile (Chelicerata, Scorpiones). Boletín de la Sociedad de Biología de Concepción (Chile) 54: 43-70.

CEPEDA-PIZARRO J (1995) Síntesis ecológica del desierto costero Peruano-Chileno. Investigación y Desarrollo (Chile) 1: 4-14.

CEPEDA-PIZARRO J, J PIZARRO-ARAYA \& H VÁSQUEZ (2005a) Composición y abundancia de artrópodos del Parque Nacional Llanos de Challe: impactos del ENOS de 1997 y efectos del hábitat pedológico. Revista Chilena de Historia Natural 78: 635-650.

CEPEDA-PIZARRO J, J PIZARRO-ARAYA \& $\mathrm{H}$ VÁSQUEZ (2005b) Variación en la abundancia de Arthropoda en un transecto latitudinal del desierto costero transicional de Chile, con énfasis en los tenebriónidos epígeos. Revista Chilena de Historia Natural 78: 651-663.

COWLING RM, PW RUNDEL, BB LAMONT, MK ARROYO \& M ARIANOUTSOU (1996) Plant diversity in Mediterranean-climate regions. Trends in Ecology \& Evolution 11: 362-366.

DESLIPPE RJ, JR SALAZAR \& YJ GUO (2001) A darkling beetle population in West Texas during the
1997-1998 El Niño. Journal of Arid Environments 49: 711-721.

ESRI (2002) ArcView GIS the geographic information system for everyone. Environmental Systems Research Institute Inc., Redlands, U.S.A. 340 pp.

FLORES GE \& J PIZARRO-ARAYA (2006) The Andes mountain range as a vicariant event in the Pimeliinae (Coleoptera: Tenebrionidae) in southern South America. Cahiers Scientifiques 10: 95-102.

FRANKIE GW, M RIZZARDI, SB VINSON, TL GRISWOLD \& P RONCHI (2005) Changing bee composition and frequency on a flowering legume, Andira inermis (Wright) Kunth ex DC. During El Niño and La Niña years (1997-1999) in Northwestern Costa Rica. Journal of the Kansas Entomological Society 78: 100-117.

GAJARDO R (1994) La vegetación natural de Chile. Editorial Universitaria, Santiago, Chile. 165 pp.

GASTON KJ (2000) Global patterns in biodiversity. Nature 405: 220-227.

LOURENÇO WR (1986) Les modèles de distribution géographique de quelques groupes de Scorpions néotropicaux. Comptes Rendus des Séances de la Société de Biogéographie 62: 61-83.

LOURENÇO WR (1991) Biogéographie évolutive, écologie et les strategies biodémographiques chez les Scorpions néotropicaux. Comptes Rendus des Séances de la Société de Biogéographie 67: 171190.

LOURENÇO WR (1994a) Diversity and Endemism in Tropical versus temperate Scorpion Communities. Biogeographica 70: 155-160.

LOURENÇO WR (1994b) Biogeographic patterns of tropical South American Scorpions. Studies on Neotropical Fauna and Environment 29: 219-231.

LOURENÇO WR (1995) Considerations sur la morphologie, écologie et biogéographie de Caraboctonus keyserlingi Pocock (Scorpiones, Iuridae). Boletín de la Sociedad de Biología de Concepción (Chile) 66: 63-69.

LOURENCYO WR (2001) On the taxonomy and geographic distribution of the genus Centromachetes Lönberg, 1897, with a redescription to Centromachetes pocockii (Kraepelin, 1894) (Scorpiones, Bothriuridae). Entomologische Mitteilungen aus dem Zoologischen Museum Hamburg 13: 305-313.

LOURENÇO WR (2002) Scorpions of Brazil. Les editions de l'If, Paris. 307p.

LOURENÇO WR (2005) Confirmation de la présence de la famille des Buthidae C.L. Koch, 1837 au Chil (Chelicerata, Scorpiones). Boletín Sociedad Entomológica Aragonesa 37: 109-112.

LOWE G \& V FET (2000) Family Bothriuridae Simon, 1880. En: Fet V, WD Sissom, G Lowe \& ME Braunwalder (eds) Catalog of the Scorpions of the World (1758-1998): 17-53. The New York Entomological Society, New York, USA. 690 pp.

MARQUET PA, F BOZINOVIC, GA BRADSHAW, C CORNELIUS, H GONZÁLEZ, JR GUTIÉRREZ, E HAJEK, JA LAGOS, F LÓPEZ-CORTÉS, L NÚÑEZ, EF ROSELLO, C SANTORO, H SAMANIEGO, VG STANDEN, JC TORRESMURA \& FM JAKSIC (1998) Los ecosistemas del Desierto de Atacama y área andina adyacente en el norte de Chile. Revista Chilena de Historia Natural 71: 593-617.

MATTONI CI (2002a) La verdadera identidad de Bothriurus vittatus (Guérin-Méneville, 1938) (Scorpiones, Bothriuridae). Revue Arachnologique 14: 59-72. 
MATTONI CI (2002b) Bothriurus picunche sp. nov., a new scorpion from Chile (Bothriuridae). Studies on Neotropical Fauna and Environment 37: 169-174.

MATTONI CI (2002c) Bothriurus pichicuy, nuevo escorpión chileno del grupo vittatus (Scorpiones, Bothriuridae). Iheringia serie Zoología 92: 81-87.

MATTONI CI (2003) Patrones evolutivos en el género Bothriurus (Scorpiones, Bothriuridae): Análisis filogenético. Tesis Doctoral, Facultad de Ciencias Exactas, Físicas y Naturales, Universidad Nacional de Córdoba, Córdoba, Argentina. i-vii + 249 pp.

MATTONI CI \& LE ACOSTA (2006) Systematics and distribution of three Bothriurus species (Scorpiones, Bothriuridae) from central and northern Chile. Studies on Neotropical Fauna and Environment 41: 235-250.

MAURY EA (1976) Escorpiones y escorpionismo en el Perú. V. Orobothriurus, un nuevo género de escorpiones altoandino (Bothriuridae). Revista Peruana de Entomología 18: 14-25.

MUÑOZ M, H NÚÑEZ \& J YÁÑEZ (1996) Libro rojo de los sitios prioritarios para la conservación de la diversidad biológica en Chile. Ministerio de Agricultura, Corporación Nacional Forestal, Santiago, Chile. 203 pp.

OCHOA JA (2004) Filogenia del género Orobothriurus y descripción de un nuevo género de Bothriuridae (Scorpiones). Revista Ibérica de Aracnología 9: 4373.

OCHOA JA (2005) Patrones de distribución de escorpiones de la región andina en el sur peruano. Revista Peruana de Biología 12: 49-68.

OJANGUREN-AFFILASTRO AA (2002) Nuevos aportes al conocimiento del género Brachistosternus en Chile, con la descripción de dos nuevas especies (Scorpiones, Bothriuridae). Boletín de la Sociedad de Biología de Concepción (Chile) 73: 37-46.

OJANGUREN-AFFILASTRO AA (2003) Un nuevo Orobothriurus (Scorpiones, Bothriuridae) de la región de Atacama, Chile. Revista Ibérica de Aracnología 7: 117-122.

OJANGUREN-AFFILASTRO AA (2004) Un nuevo Brachistosternus Pocock 1893 del norte de Chile (Scorpiones, Bothriuridae). Revista Ibérica de Aracnología 10: 69-74

OJANGUREN-AFFILASTRO AA (2005) Notes on the genus Brachistosternus (Scorpiones, Bothriuridae) in Chile, with the description of two new species. Journal of Arachnology 33: 175-192.

OJANGUREN-AFFILASTRO AA \& CI MATTONI (2006) A new species of Brachistosternus from Chilean central Andes (Scorpiones: Bothriuridae). Studies on Neotropical Fauna and Environment 41: 79-85.

PASKOFF R \& H MANRÍQUEZ (2004) Las Dunas de las Costas de Chile. Ediciones Instituto Geográfico Militar, Santiago, Chile. 112 pp

PERETTI AV (1997) Relación de las glándulas caudales de machos de escorpiones (Scorpiones, Bothriuridae) con el comportamiento sexual. Revue Arachnologique 12: 31-41.

PERETTI AV (2001) Patrones de resistencia femenina y respuesta del macho durante el apareamiento en escorpiones Bothriuridae y Buthidae: ¿qué hipótesis puede explicarlos mejor? Revista de Etología (Brasil) 3: 25-45.

PIZARRO-ARAYA J \& V JEREZ (2004) Distribución geográfica del género Gyriosomus GuérinMéneville, 1834 (Coleoptera: Tenebrionidae): una aproximación biogeográfica. Revista Chilena de Historia Natural 77: 491-500.
POLIS GA (1980) Seasonal patterns and age-specific variation in the surface activity of a population of desert scorpions in relation to environmental factors. Journal of Animal Ecology 49: 1-18.

POLIS GA (1990) Ecology. En: Polis GA (ed) The Biology of Scorpions: 247-293. Stanford University Press, California, USA. 587 pp.

POLIS GA (1993) Scorpions as model vehicles to advance theories of population and community ecology: The role of scorpions in desert communities. Memoirs of the Queensland Museum 33: 401-410.

PRENDINI L (2001) Substratum specialization and speciation in southern African scorpions: the Effect Hypothesis revisited. En: Fet V \& PA Selden (eds) Scorpions 2001. In Memoriam Gary A. Polis: 113138. British Arachnological Society, Burnham Beeches, Bucks, United Kingdom. 450 pp

PRENDINI L (2003) A new genus and species of bothriurid scorpion from the Brandberg Massif, Namibia, with a reanalysis of bothriurid phylogeny and a discussion of the phylogenetic position of Lisposoma Lawrence. Systematic Entomology 28: 149-172.

RAU JR, C ZULETA, A GANTZ, F SÁIZ, A CORTÉS, L YATES, AE SPOTORNO \& E COUVE (1998) Biodiversidad de artrópodos y vertebrados terrestres del Norte Grande de Chile. Revista Chilena de Historia Natural 71: 527-554.

ROIG-JUÑENT S \& GE FLORES (2001) Historia geográfica de las áreas áridas de América del Sur Austral. En: Llorente-Busquets J \& JJ Morrone (eds) Introducción a la biogeografía en Latinoamérica: Teorías, conceptos, métodos y aplicaciones: 257-266. Las prensas de Ciencias, Facultad de Ciencias, UNAM, México, D.F. 277 pp.

ROIG-JUÑENT S, MC DOMÍNGUEZ, GE FLORES \& CI MATTONI (2006) Biogeography history of South America arid lands. A view from its arthropods using TASS analysis. Journal of Arid Environments 66: 404-420

RUNDEL P, MO DILLON, B PALMA, HA MOONEY, SL GULMON \& JR EHLERINGER (1991) The Phytogeography and Ecology of the Coastal Atacama and Peruvian Deserts. Aliso 13: 1-50.

SAN MARTÍN P \& T CEKALOVIC (1968) Escorpiofauna chilena. I. Bothriuridae. Una nueva especie de Urophonius para Chile. Investigaciones Zoológicas Chilenas 13: 81-100.

SHMIDA A (1985) Biogeography of the desert flora. En: Evenari AM, I Noy-Meir \& DV Goodall (eds) Hot deserts and arid shrublands: 23-77. Elsevier, New York, New York, USA. 458 pp.

SISSOM WD (1990) Systematics, biogeography, and paleontology. En: Polis GA (ed) The Biology of Scorpions: 64-160. Stanford University Press, Stanford, California, USA. 587 pp.

SISSOM WD \& V FET (2000) Family Iuridae Thorell, 1876. En: Fet V, WD Sissom, G Lowe \& ME Braunwalder (eds) Catalog of the Scorpions of the World (1758-1998): 409-423. The New York Entomological Society, New York, New York, USA. 690 pp.

SQUEO FA, LA CAVIERES, G ARANCIO, JE NOVOA, O MATTHEI, C MARTICORENA, R RODRÍGUEZ, MTK ARROYO \& M MUÑOZ (1998) Biodiversidad de la Flora Vascular en la Región de Antofagasta, Chile. Revista Chilena de Historia Natural 71: 571-591.

STATSOFT, INC (2001) STATISTICA (data analysis software system), version 6. www.statsoft.com. 
TER BRAAK CJF (1995) Ordination. In: Jongman RHG, Ter Braak CJF \& OFR Van Tongeren (eds) Data Analysis in community and landscape ecology: 91169. Cambrige University Press, Cambrige, United Kingdom. 299 pp.

VIDIELLA PE, JJ ARMESTO \& JR GUTIÉRREZ (1999) Vegetation changes and sequential flowering after rain in the southern Atacama Desert. Journal of Arid Environments 43: 449-458.

Editor Asociado: Audrey Grez

Recibido el 19 de enero de 2006; aceptado el 18 de agosto de 2006
WARBURG MR \& GA POLIS (1990) Behavioral responses, rhythms and activity patterns. En: Polis GA (ed) The Biology of Scorpions: 224-246. Stanford University Press, California, USA. 587 pp. WILLIAMS SC (1987) Scorpion Bionomics. Annual Review of Entomology 32: 275-295. 
